\title{
La rénovation des groupes bulbes de l'usine marémotrice de la Rance
}

\author{
par Alain Naudy
}

EDF (USI FST)

Mulhousc Hydraulique Services

Après 30 ans de fonctionnement satisfaisant, il est devenu nécessaire de procéder à une révision générale et préventive des 24 groupes bulbes el de leur environnement.

Cette révision, pour ee qui concerne less éléments princi. paux que sonl less machines, permet par les démontages et remontages :

- de vérifier l'état gúnéral des turbines et des altemateurs,

... de détecter et de corriger d'éventuels défauts répélitifs,

- de pallier l'usure des pièces par changement ou réparation,

- de rénover les étanchéités air, huile el eau,

... d'assurer la mainlenance ou la reconstruction des alternateurs,

.- de réfléchir el d'améliorer les procédures de démontagge et de remontage alin de gagner du temps sur les indisponibilités,

- de participer à un retour d'expérjence général per metlant d'améliorer nos connajssances dans la maintenance et l'exploitation des groupes bulbes en eau de mer.

Cette maintenance de l'usine s'étend égalenent aux services auxiliaites a]tematifs of continus, aux transformateurs de puissance, aux automatismes, aux vannes du barrage,...

Ia décision a été prise d'utiliser les compétences recon nues de l'entreprise EDF dans le domaine de la maintenance des turbines hydrauliques, et en particulier des groupes bulbes.
L.entreprise EDF s'est associée aux entreprises françaises spéciałistes de l'hydroélectricité ct capables de l'aider dans cette mission. On peut citer parmi ces entreprises :

- Neyrpic à Grenoble pour les butées et la pivoterie des turbiries,

- Sarelem à Nantes pour la reconstruclion des alternateurs,

- GEC: Alsthom à Belfort pour les expertises des jantes rotor.

EDF USI EST "Mulhouse Hydraulique Services" at mis ses compétences acquises sur les groupes bulbes du Rhin, au service d'ŁDH Energie Ouest, maîlre d'ouvrage responsable de l'usine marémotrice de La Rance. L'USI Est s'appuic sur les conseils des experts hydrauliciens d'EDF, particulièrement ceux situés à lá Division Technique Générale de. Grenoble.

L'équipe d'EDF USI Est s'installe progressivement sur le chanticr de I.a Rance. Fille comporte :

- un Chef de projet chargé de la coordination générale du charıtier de maintenance vis-à-vis des différents intervenants de l'EDF et des cntreprises associées,

- un responsable mécanique chargé d'animer el de coordonner l'action des équipes locales qui effectuent les détnontages el les remontages,

... 3 équipes de 13 mécaniciens qui s'installent pro. gressivement ct dont chacune est chargée de la rénovation d'une machine à la fois.

Un compromis a élé trouvé entré:

\begin{tabular}{|l|c|c|c|c|c|c|c|c|c|c|c|c|c|}
\hline année & 1994 & 1995 & 1996 & 1997 & 1998 & 1999 & 2000 & 2001 & 2002 & 2003 & 2004 & Total \\
\hline Nb de groupes & 1 & 1,1 & 1,3 & 1,9 & 2,7 & 3 & 3 & 3 & 3 & 3 & 1 & 24 \\
\hline
\end{tabular}

Tableau 1. Planning général des opérations de rénovation de l'usine de la Rance. 


\begin{tabular}{|l|c|c|c|c|c|c|c|c|c|c|c|c|c|}
\hline année & 1994 & 1995 & 1996 & 1997 & 1998 & 1999 & 2000 & 2001 & 2002 & 2003 & 2004 & Fotal & $\vdots$ \\
\hline Nb de groupes & 1 & 1,1 & 1,3 & 1,9 & 2,7 & 3 & 3 & 3 & 3 & 3 & 1 & 24 \\
\hline coût (MF) & 12 & $\vdots$ & 15 & 33,1 & 29,9 & 38,7 & 39,1 & 39,1 & 39,1 & 39,1 & 39,1 & 25,3 & 349,5 \\
\hline
\end{tabular}

Tableau 2. Coûts totaux prévus pour la rénovation des turbines et des alternateurs.

- la nécessité d'eftectuer la totalité de la maintenance de l'usine dans un temps raisonnable du point de vue des indisponibilités,

- le dimenisonnement des équipes de maintenance,

- l'étalement de la charge financière des travaux.

Ce compromis a condujt à prévoir la totalité de la rénovaLion de l'usine sur 11 ans $\mathrm{cl}$ à passer progressivement d'un groupe rénové par an à 3 groupes rénovés pas an. Le piann ning général cies opérations est donné dans te tableau 1.

La durée globale du démontage, de la rénovation et du remontage dune machine (urbine et atternateur) dure ectuellement 13 mors et on prévoil à terme, après optimisation, une durée moyenre de 11 mois.

La surfuce utile de stockuge et les moyens de levage de l'usine ne permetent qu'une maintenance courante et le dénontage d'une mathine à la fois. Il a été nécessture de prévoir la construction d'un bâtiment de démontage et de stockage supplémentaire où seront amenés des éléments des turbines et des afternateurs. Un pont roulant principal levant 100 tonnes permettra las gros démontages-remontages des turbines ainsi que les manutentions des altcmateurs.

Ce bâtiment restera ensuite utilisable en cas de rénovation partielle de la cenlrale el pour une future grande opération de rénovation.

Ce bâtiment, don la construction débutera en 1997 , se trouverá à moins de $2 \mathrm{~km}$ de l'usine.

- En Francs 1996, les coûts toraux prévus pour la rénovation des turbines et des alterateurs, sort dornés dans le tableau 2.

L'opération de mäintenance de l'usine marémotrice de Lä Rance constitue dans sa globalité une aflaire importante di point de vue des ressources bumajnes el financières mises en jee. Elle permettra aux différents intervenants de capitaliser une expérjence dans le domaine de la maintenance des centrales marénotrices équipées de groupes bulbes. 


\section{Discussion de la journée du 21 novembre 1996}

\section{Discussion de la communication de M. Cazenave}

\section{FNTERVFrTION dN M. SOLIL,}

Dans les śconont:cs re:atives aux groupcs bubes, it faul aussj réaliser las moinde profor.deur de la fouil:e à réaliser.

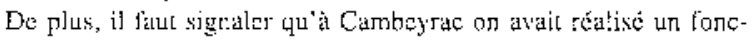
tionnetsert de l'atexnt vers '’a'al et : de balardáax.

L'usine de Cambeyrac contaît un fonctionnemare pius ou moins correcl.

\section{MALRIN}

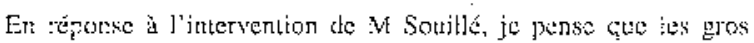
amćnagemeńls ayar: ćté réałtjsés, les chutes de petite puissarnce g: j

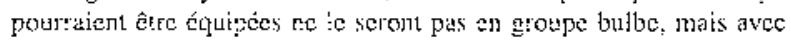

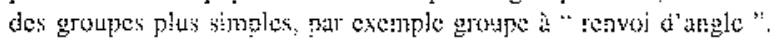

\section{Discussion de la communication de M. Bosc}

\section{AVthan}

Pensez-yots gu'j] seratil inléressant ćconomiyuener:!, t'adapter

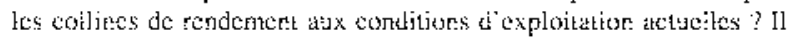
atpparat! cr ettel que pour les gropes existanls, une rénovetion des groupes (rélabilitation) pourajl representer un chalnp d'activité importiat?

\section{Réponse}

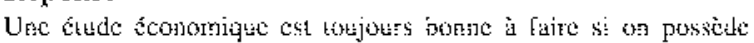
les ovtils adéchats. Il se irouve cute c'est l’énergic la plus rentable. A insi c: Corśc on assiste à une rébabilitation be la puiswance.

La question de l’amétioralion des caracértistiques bydrauliques a ćlé albordúc à plusjedrs reprises au cours de la journée du 21 rovembre. La quesijon a écé posće er ces terrnes pals M. Avetlan : " pourquoí,

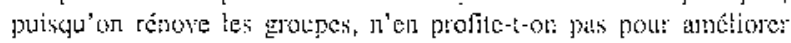
les caractéristiques de la colline au corty" " Le tracé des pales à double cambrise a été rois au point pour permelt:c, l'ostertion de

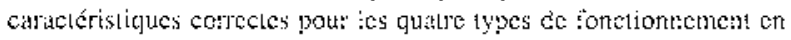

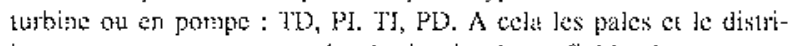
buteur permettert ine techno:ogie simple el fiable des orentes

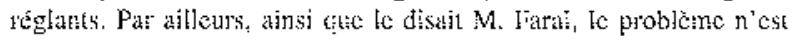

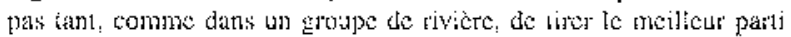
du débia maturel qui se préscntc à l'amon des otvrages, mais pluiô d'uliliser du mieux le riveás jmposé par la matréc côté me: N'oublions pas que nous tisposons d'un excédett du débil de lat ma:će jar apport au débil d’équjpement des groupes.

\section{Frark Latog:}

Quet esi l'uvenir du muliplicateu dans la conception des groupes bulbes er tes avantages potenticts sur a tajlle globale des aménagements?

\section{Réponse}

la solution actuel:e es: "'adaptation avec le multipticaticur, et ce toujours dass te condust inystadique existant, le groipe avec pate présentar. toujours la même face active. Le mécanisme de com:mande des pates es: trop complexe poir être expliqué.

\section{Discussion de la communication de M. Banal}

\section{Soliflut}

Pour compléter "intervention précécente jajoule que le cô̂t prévu de l'usine r’a pas été dépassé et constitue un des úméners economiques favo:able a prix da $\mathrm{kW}$ h.

\section{Discussion de la communication de M. Charon}

\section{M, PIAT}

L.'auto:natisation complète th: système de conciuite n'a pu se fairc çue gyấce à l'activité d'obscryation: des exploitants sur une période de 6 mois environ. Its ont ators comparé la manicte de conơire dt

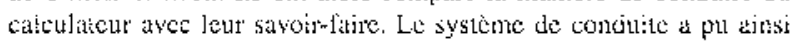

Intégrer la manjère de faje ces exploitants dans les marées te morte can an particulier el rempitr ainsi son :ôle à lon\%.

L'atomatisation te l'usjese pose le problène cic la perte de com-

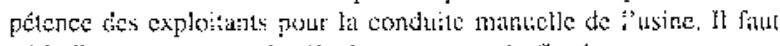

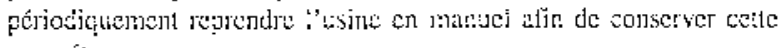
compéterice.

\section{Discussion de la communication de M. Iambert}

\section{Frrasass:}

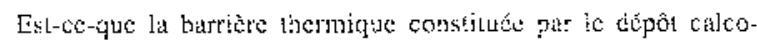

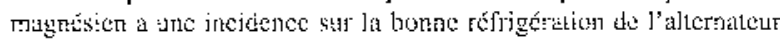
it travers :at carcasse?

\section{Réponse}

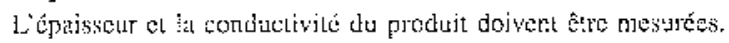

\section{Discussion de la communication de M. Salvi}

\section{MAvis}

Les premicrs alternatedas is la Rance oni tenl $: 0$ ans (1966/1976), ícs alterhatedrs rénovés ont tenu envigron 20 ans. Apris

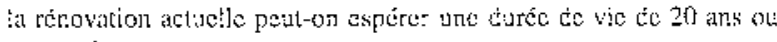
30 ats.s?

\section{Réponse}

40 ants strmblent être unc bonnc estimittion.

\section{Discussion de la communication de M. Granier}

\section{Masson}

I_es p̣hé:unènes constatés sur les butbes de la Rarece ont-ils úté rẹtrouvés sur les bulbes ctu Rhin el cu Rhône?

\section{Réponse}

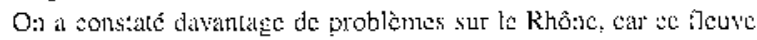
est flus puissant.

\section{Discussion de la communication de M. Naudy}

\section{DLPOR'}

En réponse à l'intervention de M, Avellar (çui prélenditil qu'i]

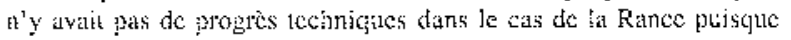

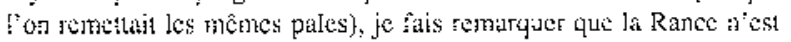
pas unc centa!e classique. I.es machines y sont soumiscs très fréGuenument à des tégínes anorndux et non siationaties. Le semplacoment des pales actueltes par des pales d’ur. nouveal tracé (er: vue d'us: ćventuel gain de productivité) nous privera:t d'un retour d exọcrience de 30 an:s qui a démontré entre actres que :'inévitab:c

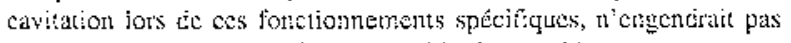
de domimages altx peles ai at l'ensembte des machines.

\section{IAHiR}

Ap:ès les incidents des alternateurs de la période de $1975 / 1982$, le lurbinage inverse a tế nís en calise. 0 : a done décidé dé ne plus fonctionner dans ce régime el bin calcul de nouveau profi] de pales

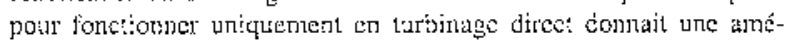
liorktion de rendement de piusteurs points. Entre lemps la cause des incidents a été trouvéce et le fonctionnement ch turbinage inverse n'a pas permis de donner suitc à ces nouvelles pirtes.

\section{Delirefil.}

Porir un reprofilage des pales, quel ess le gajn qui peul être attendu ? Quẻ cst lc coûl ç૮ ce geme d'opération ?

\section{Réponse}

Le rendement cst plus faible, majs unc sur-puissance (10 à 15\%) a peimis de s'atapter aux rouvealix crič̀tes.

Synthèse effectuée par Nelly RIOM, Sccrétaire de súance 